\title{
The use of a distractor-assigned response slows later responding in a location negative priming task
}

\author{
ERIC BUCKOLZ and ADAM GOLDFARB \\ University of Western Ontario, London, Ontario \\ and \\ MICHAEL KHAN \\ University of Wales, Bangor, Wales
}

\begin{abstract}
Responding to a target's location takes longer when that location has recently contained a distractor event (ignored-repetition [IR] trial) relative to when it has been unoccupied (control trial). This is known as the location negative priming (NP) effect. We aimed to determine whether the elevated reaction time observed for IR trials was due to the reuse of a distractor location (location locus) and/or to the need to execute a (just inhibited) distractor response (response locus). We isolated these loci latency effects by using many-to-one and one-to-many location-response assignments. Our results showed that reusing a distractor location hastened target processing at that position (facilitative location locus), whereas the production of a distractor response was associated with a time cost (interfering response locus). Accordingly, part of the latency elevation seen with IR trials results from the need on these occasions to execute a just inhibited (distractor) response, and, hence, the location NP effect has a response locus.
\end{abstract}

When individuals are required to respond on the basis of a target object's position in space, they do so more slowly when the target appears at a location that has just contained a distractor event than when the target arises at a location that has recently been unoccupied (see, e.g., Buckolz, Boulougouris, \& Khan, 2002; Buckolz, Van Damme, \& O'Donnell, 1998; Christie \& Klein, 2001; Neill, Terry, \& Valdes, 1994; Tipper, Brehaut, \& Driver, 1990). This latency difference defines the location negative priming (NP) effect. In the vernacular of the traditional location NP laboratory procedure, prime and probe trials refer, respectively, to the first and second presentations of a trial pair, each of which contains target and/or distractor events. Ignored repetition (IR) trials arise when the probetrial target stimulus appears at the prime-trial distractor location, whereas control trials refer to those occasions on which none of the prime-trial locations is reused on the probe trial.

On the basis of arguments made elsewhere (Buckolz, Boulougouris, \& Khan, 2000; Tipper, 2001; Tipper, Meegan, \& Howard, 2002), we decided to adopt, without prejudice, some of the tenets of the inhibition-based explana-

This work was supported by a grant from the Natural Sciences and Engineering Council of Canada to E.B. We are indebted to Tram Neill for several instructive comments. We also thank Ioan Todoran for programming the procedures used here and Sarah Guy for her assistance with the data analyses. Correspondence concerning this article should be addressed to E. Buckolz, School of Kinesiology, Faculty of Health Sciences, Thames Hall 4150, University of Western Ontario, London, ON, N6A 3K7 Canada (e-mail: ebuckolz@uwo.ca). tion of the NP effect (described below) as a framework within which to set out the objectives of the present research and as a basis for interpretation of the results obtained (Houghton \& Tipper, 1994).

A fundamental component of the inhibition-based model is that the NP process begins with the active inhibition of the distractor event during prime-trial processing and that it is the persistent aftereffects of this inhibition that carry forward and act to delay probe-target processing when it appears at the prime-trial distractor location (i.e., IR trial; Tipper, 1985; Tipper \& Cranston, 1985). It is not clear, however, whether the inhibition directed toward the prime-trial distractor event is applied to an internal representation of the distractor's location (i.e., a location locus), to its assigned response (i.e., a response locus), or to both. Because both the prime-trial distractor location and its associated response are reinvolved on IR trials (i.e., the probe target occupies the prime-trial distractor location, thereby requiring the execution of the prime-trial distractor response [DR]), either of these two loci could be responsible for the lengthened reactions seen on IR trials. By extension, either locus could cause the location NP effect. Given the virtual absence of prior work on the locus question, the fundamental objective of this investigation was to determine whether a response locus made a contribution to the production of the location NP effect. The possibility of a location locus will also be examined, but less extensively.

Despite the shortage of direct evidence, a consensual opinion seems to have developed, according to which the NP effect has a location locus (see, e.g., Baylis, Tipper, 
\& Houghton, 1997; Christie \& Klein, 2001; Milliken, Tipper, Houghton, \& Lupiáñez, 2000). According to Baylis et al. (1997), the effective locus of prime-trial distractor inhibition is determined by the target feature that controls correct response selection. In a location task, this would be the prime-trial distractor's spatial position (i.e., location locus). Presumably, when a distractor event appears at a location, the location's representation is inhibited so as to discourage the future processing of events that later arise at that position in space.

In fact, the research used to promote a location locus for the NP effect for location tasks consists of a single study (Neill, Valdes, \& Terry, 1995). Neill et al. (1995) mapped two locations onto each of two possible keypress responses (4-to-2 location-response assignment) and used target-only probe trials. The critical aspect of this procedure was that it allowed the probe target to appear at an unused prime-trial location while requiring the use of the prime-trial distractor response (distractor-response repetition [DRR] trial). The contribution of reusing the primetrial distractor location to reaction time (RT) (i.e., a location locus) could then be determined by contrasting the latencies obtained for the DRR trials with those obtained when both the prime-trial distractor location and its response were reinvolved on the probe trial (i.e., the traditional IR trial). Neill et al. (1995) found that RT was longer for the IR than for the DRR trials. They concluded that reusing a prime-trial distractor location slowed processing of the probe target when it appeared there, thus contributing to the prolonged RTs seen with IR trials and, hence, to the production of the location NP effect (i.e., a location locus).

We are unaware of any published work on a possible response locus for the location NP effect (the study of Neill et al., 1995, was not designed to test this possibility). There is, however, sufficient indirect evidence to justify the expectation of a response locus contribution to the location NP effect and so to warrant its examination here.

For example, it has been well established on the basis of electrophysiological indices reflective of output activation (e.g., electromyography, lateralized readiness potential) that irrelevant/distractor display events result in the retrieval/activation of their assigned responses (see, e.g., Coles, Gratton, Bashore, Eriksen, \& Donchin, 1985; De Jong, Liang, \& Lauber, 1994; Eriksen, Coles, Morris, \& O'Hara, 1985; Smid, Mulder, \& Mulder, 1990; ValleInclán \& Redondo, 1998). Presumably, the latency elevations (interference) produced when these activated DRs appeared was the result of the need to inhibit these incorrect output urges (i.e., the "late" filter; Deutsch, Deutsch, Lindsay, \& Treisman, 1967). With regard to location NP tasks, it seems reasonable to expect that the appearance of a distractor event at a prime-trial location would lead to the automatic activation, and subsequent inhibition, of the location's assigned response. Possibly, the need to use an inhibited DR on IR trials requires that the interfer- ing effects that result from its earlier inhibition be set aside before initiation can take place. This may take time, which would account for the longer RTs found for IR trials. Since IR trial latency is used to calculate the location NP effect, it would be clear that the NP effect would owe some of its existence to a response locus.

A response locus for the location NP effect is also suggested by recent findings obtained outside of the NP paradigm. Eimer, Schubo, and Schlaghecken (2002, Experiment 1) provided their participants with a centrally located, uninformative cue (an arrowhead) that pointed to the left or to the right, which they immediately masked with a $100-\mathrm{msec}$ double-arrowhead presentation. In one condition, the target event, which followed the mask offset, was also a centrally positioned arrowhead, and the participants were to respond with a left- or right-hand buttonpress compatible with the direction pointed to by the target. Eimer et al. found that buttonpress RTs were longer if the prime and the target arrowheads pointed in the same direction rather than in different directions (i.e., a negative compatibility effect). On the basis of this finding, Eimer et al. claimed that their masked cue led to the activation (see Eimer, 1999, for electrophysiological support) and subsequent inhibition of its assigned response. The slower responding that they found with the compatible trials thus reflected the fact that the use of a just inhibited output takes longer to initiate than the use of one that has not been recently suppressed. If the prime-trial distractor location in NP tasks acts like the masked cue used by Eimer et al., the location NP effect ought to have a response locus component. Again, this is because IR trials, used to index the location NP effect, require the use of a just inhibited prime-trial DR. The caveat here is that Eimer et al. used neither an NP design nor a location task and so the applicability of their data to the location NP effect is not direct.

From a design perspective, our examination of the locus question related to the location NP effect included both many-to-one $(2: 1)$ and one-to-many (1:2) locationresponse mappings, along with the traditional one-toone assignments. This was accomplished by using five horizontally aligned event locations (see Figure 1).

The critical trials are those in which the prime-trial distractor appeared at L3, followed by the delivery of the probe target at either L2 or L4 (i.e., DRR trials; see Figure 2A). The assumption here, supported by Eimer et al. (2002), was that both of the responses assigned to L3 would be activated and then inhibited when the primetrial distractor appeared there. The appearance of the probe target at either L2 or L4 would then necessitate the use of the just inhibited prime-trial DR but would not entail the reuse of the distractor's location. Evidence of a response locus for the NP effect would take the form of a mean latency for these DRR trials in excess of that of a control condition, in which neither the probe target location nor its response was used on the prime trial (Figure $2 \mathrm{~A}$. vs. 2B). On the other hand, a location locus simi- 
lar to that found by Neill et al. (1995) would be indicated if the latency for the IR trials exceeded that found for the DRR trials (Figure $2 \mathrm{C}$ vs. 2A).

The data of the internal trials, in which the probe target appears at L3, were also a potential source of support for a response locus on two accounts. First, DRR versus control-trial latency contrasts will be possible for selfselected responses (Figure 2D vs. 2E). Slower responding for the DRR than for the control trials would again be in line with the idea that the use of a just inhibited response delays processing, which, in turn, would point to the involvement of a response locus contribution to the location NP effect. Second, on a portion of the internal trials, the choice of response will involve a selection between a prime-trial DR and a control response (Figure 2D). Should the latency analyses show that the enforced use of DRs slow subsequent processing, it would be interesting to see if participants, perhaps recognizing these deleterious effects, would show a tendency to avoid prime-trial DR usage.

\section{METHOD}

\section{Participants}

Twenty-three right-handed university students ( 11 males, 12 females) with normal or corrected-to-normal vision volunteered to participate in this study.

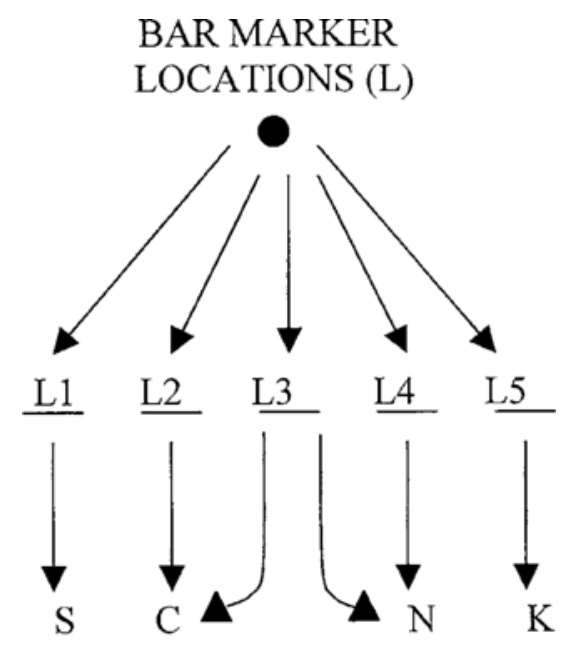

(keyboard button responses)

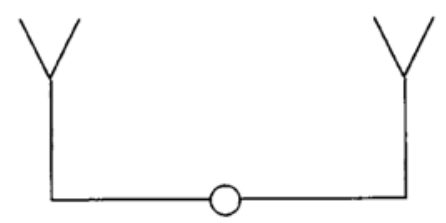

Schematic Figure

Figure 1. Schematic representation of the location-response assignments.

\section{Apparatus}

The input display was presented on a $47.5-\mathrm{cm}$ computer screen situated on a tabletop located $73.5 \mathrm{~cm}$ above the floor and $198 \mathrm{~cm}$ from the participant. Five horizontally arranged bar markers, $1.3 \mathrm{~cm}$ in length and $0.9 \mathrm{~cm}$ apart, appeared in the middle of the computer screen to denote the potential locations for delivery of the target and/or distractor events (to facilitate the exposition of the method employed, these bar marker locations are numbered L1-L5, from left to right; see Figure 1). The horizontal distance between the bar markers for L1 and L5 was $8.8 \mathrm{~cm}$ center to center, subtending an approximate visual angle of $2.4^{\circ}$. The target stimulus and the distractor events were the same shape (rectangles) and size $(1.8 \mathrm{~cm} \times 1.2 \mathrm{~cm})$, but they differed with respect to color, the target being light blue and the distractor dark blue.

In order to respond to the appearance of a target stimulus, the participants sat with their forearms placed on a desk top, with the third digit and index finger resting on keyboard buttons " $\mathrm{S}$ " and " $\mathrm{C}$," respectively (left hand) and " $\mathrm{K}$ " and "N," respectively (right hand; see Figure 1). These button responses were assigned to bar marker Locations L1, L2, L4, and L5, respectively, and were to be depressed through a finger flexion action dependent on the location assumed by the target stimulus. When the target appeared at (i.e., slightly above) bar marker Location L3, the participants were free to respond by pressing either "C" or "N." In keeping with Baylis et al. (1997), these trials are called internal trials, whereas external trials refer to those occasions when the target event appeared at bar marker Locations L1, L2, L4, or L5. Also appearing on the computer screen, below the bar markers, was a schematic or stick figure (overhead view) of a participant, as is shown in Figure 1 (see Buckolz, Boulougouris, O'Donnell, \& Pratt, 2002, for further details). Basically, the lines delineating the forearms ended in Vs, intended to represent the third digit and index finger of the left and right hands. These "digits" extended to within $1 \mathrm{~cm}$ of the bar markers to which they were respectively assigned for the external trials. This stick figure was included with the aim of reducing any confusion that might arise, due to the long viewing distance, as to which locations were being occupied by the display events delivered.

\section{Procedure}

It was emphasized that trials would be presented in pairs: first a prime trial and then a probe trial. Each pair of trials commenced with a 100 -msec auditory click (warning signal) followed $500 \mathrm{msec}$ later by the prime-trial display events, which always consisted of both a target and a distractor. Upon initiation of the correct response, the prime display events disappeared from the screen and were replaced $500 \mathrm{msec}$ later by the probe items, which contained either a target with a distractor or a target alone. With the execution of the correct probe output, the display events were once again removed. Following an intertrial interval of $1,500 \mathrm{msec}$, the warning click was delivered, signaling the beginning of the next prime-probe trial pair.

The participants completed 52 prime-probe trial pairs, with trial order varying randomly between participants. A distractor event always accompanied the target stimulus on the prime trials but appeared unpredictably on about $40 \%$ of the probe trials. The location placements for the target and distractor events were selected to maximize the number of trials needed for analysis purposes, and an attempt was made to keep the frequency of probe-trial location appearance for these events close to equiprobable. In this regard, the probe target appeared at each of L1, L2, L4, and L5 on $18 \%$ of the trials administered, and at L $328 \%$ of the time. This small frequency imbalance, even if perceived, should not alter NP priming (Buckolz, Boulougouris, O'Donnell, \& Pratt, 2002). ${ }^{1}$ The probe-trial distractor event appeared with approximately equal frequency at all locations $(21 \%, 19 \%, 20 \%, 19 \%$, and $21 \%$ for L1, L2, L3, L4, and L5, respectively). 


\section{External Trials (Trial Type Examples)}

A. Distractor-response repetition trials

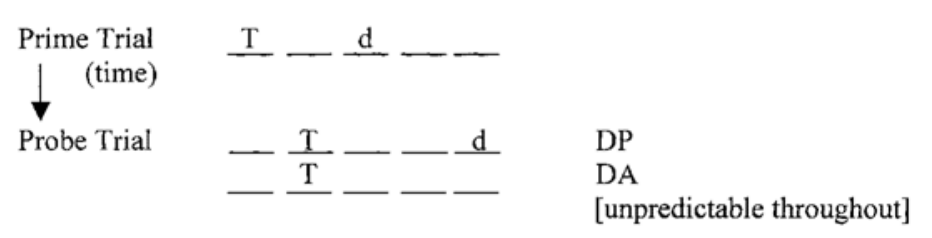

B. Control trial

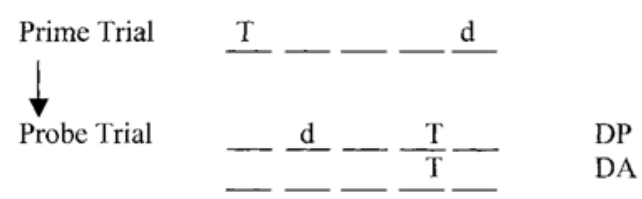

C. Ignored-repetition trial

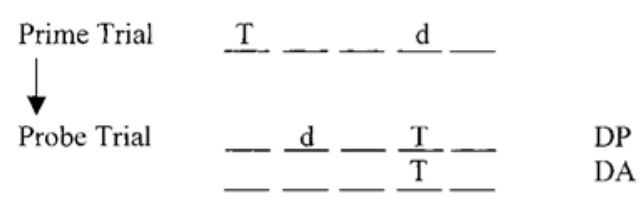

II. Internal Trials (Trial Type Examples)

D. Distractor-response/Control trial

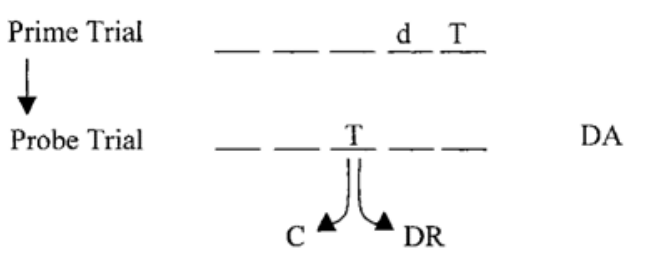

E. Control/Control trial

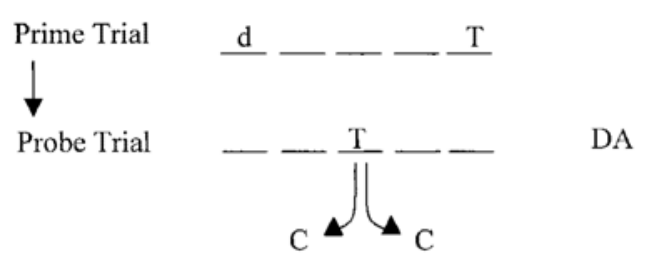

F. Ignored-repetition trial

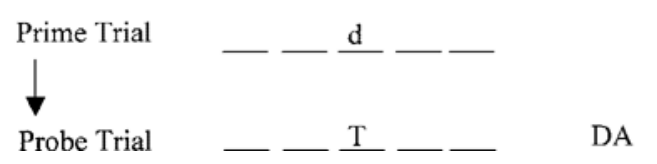

Figure 2. Exemplars of the critical external and internal trial types. T, target; d, distractor; DP, distractor present; DA, distractor absent. 
For the prime trials, the target appeared at L1-L5 on $33 \%, 8 \%$, $17 \%, 8 \%$, and $33 \%$ of the trials run, respectively. The frequencies of distractor appearance over these locations were $19 \%, 11 \%, 39 \%$, $11 \%$, and $19 \%$, respectively.

Overall, there were 380 external and 140 internal trials. Exemplars of the external and internal probe trial types used in the analyses are illustrated in Figure 2.

Three external trial types were of interest: (1) prime DRR trials, in which the probe target required the use of the prime-trial distractorassigned response but did not reuse the prime-trial distractor event's location (Figure 2A), and which occurred either with ( $n=40$ trials) or without ( $n=28$ trials) a probe distractor; (2) control trials, in which neither of the locations used on the prime trial was utilized on the probe trial (Figure 2B; distractor absent, $n=64$; distractor present, $n=24$ ); and (3) IR trials, in which the probe target appeared at the location that had held the prime-trial distractor (Figure 2C) and which also occurred either with $(n=24)$ or without $(n=24)$ a probe distractor.

Our interest in the internal probe trials was restricted to those occasions when the target appeared alone ( $n=96$ trials $)$ so that a distractor event could not influence probe response selection. Actual response selection on the internal trials was recorded by the computer program, which controlled the trial sequence and measured RTs. On 40 of these trials, the prime-trial distractor event occurred at either L2 or L4. On these occasions, the participants were free to choose either the prime-trial DR or a control (C) output (i.e., a response not involved on the prime trial [DR/C] trials; see Figure 2D). Also included were 40 internal trials in which the participant chose between two control outputs (i.e., $\mathrm{C} / \mathrm{C}$ trials). In these instances, the prime-trial distractor was located at either L1 or L5 (Figure 2E).

Finally, there were 16 trials in which the prime-trial distractor and the probe-trial target both appeared at L3 $(n=16$; Figure $2 \mathrm{~F})$. The remainder of the trials were not analyzed.

The participants received the following instructions and information before testing commenced: (1) They were instructed to respond to the location of the target event (light blue rectangle) as quickly as possible while ignoring a distractor item (dark blue rectangle) when present; (2) the location-response assignments were reviewed, and it was pointed out that the participants were free to use either index finger to respond when the target appeared at L3; (3) they were told to avoid errors; an error message would appear on the screen in the event of an incorrect buttonpress, and the trial sequence would resume when the participant pressed the space bar; (4) trials would be presented in pairs, the first trial in each pair following the presentation of the warning tone, with the time separating two trials within a pair being shorter than that separating between-trial pairs; (5) although the prime target would always be accompanied by a distractor on the prime trials, this would not always be the case for the probe trials; and (6) the probe target and distractor (when delivered) would appear randomly over the trial series and would occur with approximately equal frequency at all of the bar marker locations.

Following the presentation of this information, the participants completed 10 practice trials and had any resulting questions answered to ensure that they understood the task requirements. The experimental session then began with the participants completing 520 trial pairs. Two-minute rest periods were enforced after completion of the first and third blocks of 125 trial pairs, with a $15-\mathrm{min}$ break occurring when the second block of 125 trial pairs had been finished. The participants then completed the final block of trials, which consisted of 145 trial pairs. They were also allowed to request a rest at any time during a trial block, at which point the experimenter halted the session until the participant wished to continue.

\section{RESULTS AND DISCUSSION}

Response times of less than 100 msec (anticipations; $<1 \%$ ) or greater than $1,000 \mathrm{msec}$ (insufficient vigi-
Table 1

Mean Within-Participants Reaction Times (RTs, in Milliseconds) for the External Trials as a Function of Probe Distractor Condition and Probe Target Location

\begin{tabular}{|c|c|c|c|c|c|c|c|c|}
\hline \multirow[b]{4}{*}{ Trial Type } & \multicolumn{8}{|c|}{ Probe Distractor Condition } \\
\hline & \multicolumn{4}{|c|}{ Distractor Absent } & \multicolumn{4}{|c|}{ Distractor Present } \\
\hline & \multicolumn{2}{|c|}{$\mathrm{L} 2$} & \multicolumn{2}{|c|}{ L4 } & \multicolumn{2}{|c|}{$\mathrm{L} 2$} & \multicolumn{2}{|c|}{ L4 } \\
\hline & RT & $S D$ & RT & $S D$ & RT & $S D$ & RT & $S D$ \\
\hline DRR & 515 & 73 & 490 & 80 & 559 & 77 & 536 & 80 \\
\hline Control & 486 & 55 & 450 & 49 & 542 & 70 & 509 & 73 \\
\hline Response locus & $29 *$ & & $40 *$ & & $17 *$ & & $25^{*}$ & \\
\hline IR & 495 & 90 & 467 & 82 & 567 & 84 & 523 & 91 \\
\hline DRR & 515 & & 490 & & 559 & & 536 & \\
\hline Location locus & $-20 *$ & & $-23 *$ & & 8 & & -13 & \\
\hline IR & 495 & & 467 & & 567 & & 523 & \\
\hline Control & 486 & & 450 & & 542 & & 509 & \\
\hline Negative priming & 9 & & $17 *$ & & $25^{*}$ & & 14 & \\
\hline
\end{tabular}

Note-L2, nonpreferred hand; L4, preferred hand. Trial types used to calculate locus effects (response, location) were as follows: DRR, distractorresponse repetition; IR, ignored repetition; and control. Buttonpress error rates: DRR trials, $6.4 \%$ and $6.3 \%$; IR trials, $4.2 \%$, and $6.2 \%$; control trials, $-4.7 \%$ and $4.7 \%$ for the distractor-absent and distractor-present conditions, respectively. ${ }^{*} p<.05$.

lance; $<1 \%$ ), along with buttonpress errors (Table 1 ), were excluded from RT analyses. One participant's data had to be removed because of high error rates $(>20 \%)$. Only the probe-trial data are reported below.

Overall, the latency results obtained here point to the involvement of both a response and a location locus on IR trials. The pattern evident in Table 1 is that the reuse of the prime-trial distractor location facilitates processing of the probe target at this spatial position (location locus), whereas the need to execute the prime-trial DR slows response initiation (response locus). Since these two loci have opposing influences on latency, it is clear that the production of the location NP effect owes its existence to the dominating interference impact associated with a response locus. We turn now to the data.

\section{The Locus Question}

Response locus-external trials. Since the DRR trials involved only L2 and L4, the control data used for this analysis were also restricted to these two locations (Figures 2A and 2B). A three-way analysis of variance (ANOVA) was calculated using mean participant RT, with priming (DRR vs. control), probe target location (L2 vs. L4), and probe distractor condition (present vs. absent) serving as the main within-participants factors. The cell means for this analysis are presented in Table 1 .

Only the main effects of priming $[F(1,21)=14.05$, $\left.M S_{\mathrm{e}}=2,419, p<.01\right]$, probe target location $[F(1,21)=$ $\left.6.88, M S_{\mathrm{e}}=5,283, p<.03\right]$, and distractor condition $\left[F(1,21)=48.03, M S_{\mathrm{e}}=2,365, p<.01\right]$ proved to be statistically significant. As was expected, latencies were larger when a distractor accompanied the probe target $(536 \mathrm{msec})$ relative to when it did not $(485 \mathrm{msec}$; see Buckolz, Boulougouris, \& Khan, 2002; Neill et al., 1994), and RTs were longer for nonpreferred-hand (left: L2) than 
for preferred-hand (right: L4) finger responses (525 vs. $496 \mathrm{msec}$, respectively).

Most important, however, is the fact that the mean DRR latency significantly exceeded that obtained for control trials ( $525 \mathrm{vs.} 497 \mathrm{msec}$ ). Clearly, the later use of a DR slows responding. The extended RT produced on the DRR trials can be attributed only to the need to execute a distractor output, since the location component of these trial types was the same (i.e., it was unused on the prime trial). Likely, what happens is that the prime-trial DR undergoes automatic activation by virtue of an event appearing at that location (Eriksen et al., 1985; ValleInclán \& Redondo, 1998), which is followed by inhibition in order to prevent its unwanted initiation (Eimer, 1999; Eimer et al., 2002). The result of this applied (DR) inhibition is a persistent aftereffect that acts to prevent the future use of this response. Overcoming this interferencecausing aftereffect takes time, delaying target response initiation.

Because the probe target on IR trials requires the use of the prime-trial DR, elevations in IR RTs beyond that of a control condition are produced at least partly by the mandated execution of a DR (response locus). It follows that the location NP effect too has a response locus, given that this effect's presence is indexed by the latency difference between IR and control trials.

On a procedural note, we gained support for our assumption that the prime-trial distractor would lead to the activation and inhibition of both of the responses mapped onto L3 when the distractor was there. This follows from the fact that the latency difference between the DRR and control trials was obtained when the probe target appeared unpredictably at either L2 (left response) or L4 (right response). Thus, using either of the responses associated with L3 delayed probe-trial responding. This multiple response activation and subsequent inhibition, set in motion by a single input (uninformative prime-trial event), was reported by Eimer et al. (2002, Experiment 2).

Response locus-internal trials. Internal trials refer to those occasions when the probe target appeared at bar marker L3, allowing participants to choose which of two response options (i.e., left or right index finger) would be used on that trial.

The fundamental reason for examining these internal trials was to look for evidence of a response locus. This could be forthcoming in two ways. First, DRR latencies could exceed those obtained for a control condition. Second, participants could show an aversion to the use of prime-trial DRs when given the opportunity to do so (i.e., on distractor-response/control [DR/C] trials; Figure 2D), possibly in recognition of the fact that such responses have a deleterious effect on processing time. In looking into these possibilities, only distractor-absent probe trials were used so that response selection would not be influenced by the positioning of a distractor event.

Latency data. We contrasted the latencies for the DRR trials from the DR/C condition with those of the control trials obtained with the $\mathrm{C} / \mathrm{C}$ condition (Figure $2 \mathrm{D}$ vs. $2 \mathrm{E}$ ) using only dominant-hand RTs, which constituted about $80 \%$ of participant self-selections on the DR/C trials. Because all the participants showed a right-hand preference, this amounted to using only those trials in which the prime-trial distractor appeared at L4 and L1 on the $\mathrm{DR} / \mathrm{C}$ and $\mathrm{C} / \mathrm{C}$ trials, respectively. The prime target appeared at L5 in both cases. The selection and use of the prime-trial DR in the DR/C condition involved reusing the same hand on both the prime and probe trials. In order to have the control trials meet the same restriction, we had to use control trial data from the $\mathrm{C} / \mathrm{C}$ condition.

Again, it was evident that reinvolving the prime-trial DR, but not its location, significantly slowed probe responding $(\mathrm{RT}=440 \mathrm{msec}$ for DRR trials) in comparison with the control condition (422 msec), in which no aspect of the prime-trial distractor event was repeated $[t(21)=$ 2.41, $S D D=33.93, p<.05]$. This finding is consistent with that reported for the external trials and extends it by showing that the responding delays caused by the use of a DR persist even when participants self-select their use. Once more, the implication is that the location NP effect has a response locus because IR trials, used to calculate this effect, require the use of the prime-trial distractor output. As an aside, the RT slowing seen here even when DRs were self-selected runs counter to the view that the location NP process does not operate when participants have this kind of choice (Baylis et al., 1997).

Selection data. There was no indication that the participants avoided the use of the prime-trial DR when given the opportunity to do so (DR/C trials, Figure 2D), and so there was no evidence of a response locus on this account. The thinking was that individuals might refrain from selecting a DR because of knowledge that it would impede processing (response locus).

Here, we simply report that in the baseline $\mathrm{C} / \mathrm{C}$ condition (Figure 2E), in which the participants simply chose between two control responses, it is clear that the principal basis of response selection was hand preference. The participants used their preferred-hand finger response about four times more often than the nonpreferred-hand alternative (.79 vs. .21) when responding to L3 probe target placements. An identical pattern was observed for the DR/C trials (Figure 2D), indicating that the presence of a distractor-response alternative did not alter the response selection strategy for the L3 target placement. Thus, there was no evidence here showing an aversion to the use of DRs, even though the latency data reported earlier showed that the use of such outputs slows responding. Thus, no support for a response locus for the location NP effect is available on this basis.

Location Locus-external trials. An ANOVA was carried out using only the individual RT data produced with L2 and L4. Again, priming (IR vs. DRR trials), probe target location (L2 vs. L4), and probe distractor condition (present vs. absent) served as the main factors. Both the probe distractor condition $\left[F(1,21)=14.91, M S_{\mathrm{e}}=8,636\right.$, $p<.01]$ and the probe target location $[F(1,21)=7.14$, $\left.M S_{\mathrm{e}}=5,448, p=.01\right]$ produced significant main effects. 
RTs were longer when a probe distractor accompanied the target (546 vs. $494 \mathrm{msec}$ ), and preferred-hand reactions were faster than those produced with the nonpreferred hand (L4: 504 msec; L2: 534 msec; see Table 1).

The priming factor did not achieve significance $\left[F(1,21)=1.30, M S_{\mathrm{e}}=4,741, p=.26\right]$. Planned comparisons carried out for the levels of the probe distractor condition revealed a significant priming effect for the distractor-absent $\left[F(1,21)=4.30, M S_{\mathrm{e}}=3,198, p<.05\right]$ but not for the distractor-present $[F(1,21)<1]$ probe trials. For the distractor-absent probes, latencies were longer for the DRR $(503 \mathrm{msec})$ than for the IR $(481 \mathrm{msec})$ trials (as was the case for the distractor-present probes; see Table 1). These results show that repeating the prime-trial distractor location (on IR trials) facilitates rather than interferes with target processing at that spot. It seems that representations of distractor locations are free of primetrial inhibition and, thus, of any responsibility for the location NP effect.

The foregoing conclusion, and its antecedent result, are contrary to those of Neill et al. (1995), who found that the reuse of a distractor location resulted in delayed processing at that position [RT(IR) $>$ RT(DRR)]. The explanation for these discordant findings likely resides in procedural differences. One possibility is that, whereas Neill et al. (1995) also used distractor-free probe trials, unlike here, the absence of their probe distractors was predictable. According to other work, the predictable withholding of the probe distractor by Neill et al. should have resulted in a disruption of the NP process, evident in the removal of the location NP effect (Buckolz, Boulougouris, \& Khan, 2002; Guy, Buckolz, \& Pratt, 2004; Tipper et al., 1990). So, either the location NP process was not functional in the Neill et al. (1995) study despite the $\mathrm{RT}(\mathrm{IR})>\mathrm{RT}(\mathrm{DRR})$ result, or it was, but perhaps in some unusual manner, which might account for the different location locus findings they obtained.

The traditional location NP effect. For the most part, the traditional location NP effect (IR vs. control trials) was evident in our results, providing some assurance that the present procedures had not unintentionally disrupted the NP process.

External trials. An ANOVA was calculated using individual mean RTs, with priming (IR vs. control), probe target location (L2 vs. L4), and probe distractor condition (present vs. absent) acting as the main factors. RTs were reliably shorter for preferred (L4) versus nonpreferred (L2) hand conditions $\left[F(1,21)=8.99, M S_{\mathrm{e}}=5,339, p<\right.$ $.01]$, as well as when the probe trial contained a distractor in comparison with when it did not $[F(1,21)=25.33$, $\left.M S_{\mathrm{e}}=5,948, p<.01\right]$. Although an NP effect was evident for all conditions examined (Table 1), the main effect of priming lacked statistical significance $[F(1,21)=2.61$, $\left.M S_{\mathrm{e}}=5,649, p=.12\right]$. However, planned comparisons showed that an NP effect was evident on two occasions: distractor absent, preferred hand, and distractor present, nonpreferred hand $(p<.05)$.
Internal trials. Here, we looked at the location NP effect for self-selected responses (Table 2F). An ANOVA was calculated using individual mean RTs, with probe response location (L2 vs. L4) and priming (IR vs. control) serving as the main factors. The main effect of priming $(27 \mathrm{msec})$ was significant $[F(1,21)=10.72$, $\left.M S_{\mathrm{e}}=1,371, p<.01\right]$. This is reflective of the fact that RT for the IR trials $(460 \mathrm{msec})$ exceeded that obtained for the control trials (433 $\mathrm{msec}$ ), pointing to the presence of a location NP effect.

\section{SUMMARY}

The essential finding here was that the enforced or self-selected use of a prime-trial DR slowed probe RT relative to a control condition. This result is in accordance with Eimer et al. (2002) in suggesting that future efforts to execute activated yet unused (inhibited) responses will prolong processing. Since the traditional IR trial used to calculate the location NP effect includes the use of a prime-trial DR, it follows that this priming effect has a response locus. The contribution of a location locus to RT and, hence, to the location NP effect, is still unclear. Our results indicated that reusing the prime-trial distractor location reduced probe target processing time at that position, whereas Neill et al. (1995) reported the reverse. Additional work will be needed to clarify the latency impact resulting from distractor location repetition.

The discovery here that the location NP effect owes its existence in some measure to the time cost associated with the delay encountered when an attempt is made to initiate a recently inhibited response (i.e., this NP effect has a response locus) is at odds with the consensual view that this effect arises because of slowed processing taking place at a reused distractor location (location locus; Baylis et al., 1997; Neill et al., 1995). Not surprisingly, then, existing theories proffered to explain the NP effect (see Tipper, 2001) have not explicitly assigned the cause of this effect to a response locus. At least for the location variant of the NP task, which was studied here, current and future explanations of the NP effect ought to incorporate a response locus contribution.

We leave the details of this locus incorporation, if it is needed, to the producers and/or proponents of the various NP explanations. By way of a general comment, however, it seems that the accommodation of an output locus can be readily accomplished by two of the main existing NP theories. For example, inhibition-based models (see, e.g., Houghton \& Tipper, 1994) can simply contend that prime-trial distractor processing involves the inhibition of the (activated) prime-trial DR. Furthermore, the required use of this output on IR probe trials, responsible for the NP effect, necessitates a time-consuming setting aside of the delaying effects that arise as a result of the earlier inhibition applied to the prime-trial distractor output. The episodic theory (see, e.g., Neill, 1997), in contrast, does not include the notion of prime- 
trial distractor inhibition in any fashion, but it does include the idea that the prime-trial distractor output can be labeled (e.g., "do not respond"; see Klein \& Taylor, 1994) independent of location labeling and in a way that would cause a slowing of processing if the DR was used later on the probe trial (i.e., an output locus). Naturally, our findings would require that both theories discount the reuse of a distractor location (IR trial) as contributing to the location NP effect.

Finally, we simply highlight the fact that another of the major NP explanations - namely, the temporal discrimination theory (Milliken, Joordens, Merikle, \& Seiffert, 1998) - does not seem able to readily accommodate the locus findings reported here. We hastily add that this is not necessarily a problem. This is because the temporal discrimination idea was generated on the basis of data produced by the identity version of the NP procedure, and so was perhaps intended to explain only identity NP operations. If so, Milliken et al. (1998) are under no obligation to incorporate the present locus results within their theory until they are shown to hold for the identity variant of the NP task. Such a demonstration is by no means a given, since the response of the NP effect to the same independent variables has not always been the same for the location versus the identity versions of the NP paradigm (cf. Moore, 1994, vs. Buckolz, Boulougouris, \& Khan, 2002). Keeping this point in mind, we note that according to the temporal discrimination idea, the slowing produced on IR trials is held to be the result of such trials' containing both "old" and "new" (incongruent) information. With respect to the location variant of the NP procedure studied here, the repeated use of the primetrial distractor location would be old, whereas the response (i.e., "execute" rather than "inhibit") to this location would be new on probe IR trials. In plain terms, the present findings indicate that a latency slowing (i.e., the location NP effect) arises whether the prime-trial distractor location is repeated (old; IR trials) or not (new; our DRR trials) on the probe trial, as long as the prime-trial DR is needed. Thus, an incongruence between old and new on the probe trial is not required for the lengthened reactions seen on IR trials for location tasks. This seems to be at odds with the temporal discrimination theory.

\section{REFERENCES}

Baylis, G. C., Tipper. S. P., \& Houghton, G. (1997). Externally cued and internally generated selection: Differences in distractor analysis and inhibition. Journal of Experimental Psychology: Human Perception \& Performance, 23, 1617-1630.

Buckolz, E., Boulougouris, A., \& KHAN, M. (2002). The influence of probe-trial selection requirements on the location negative priming effect. Canadian Journal of Experimental Psychology, 56, 274-283.

Buckolz, E., Boulougouris, A., O’Donnell, C., \& Pratt, J. (2002). Disengaging the negative priming mechanism in location tasks. $\underline{E u-}$ ropean Journal of Cognitive Psychology, 14, 207-225.

Buckolz, E., Van Damme, J., \& O’Donnel L, C. (1998). Positive priming: Locations containing relevant information are labeled accordingly. Human Movement Science, 17, 781-799.

Christie, J., \& Klein, R. (2001). Negative priming for spatial location? Canadian Journal of Experimental Psychology, 55, 24-38.
Coles, M. G. H., Gratton, G., Bashore, T. R., Eriksen, C. W., \& DonCHIN, E. (1985). A psychophysiological investigation of the continuous flow model of human information processing. Journal of Experimental Psychology: Human Perception \& Performance, 11, 529-553.

De Jong, R., Liang, C.-C., \& Lauber, E. (1994). Conditional and unconditional automaticity: A dual-process model of effects of spatial stimulus-response correspondence. Journal of Experimental Psychology: Human Perception \& Performance, 20, 731-750.

Deutsch, J. A., Deutsch, D., Lindsay, P. H., \& Treisman, A. M. (1967). Comments on selective attention: Perception or response. Quarterly Journal of Experimental Psychology, 19, 362-367.

EIMER, M. (1999). Facilitatory and inhibitory effects of masked prime stimuli on motor activation and behavioral performance. Acta Psychologica, 101, 293-313.

Eimer, M., SCHuBo, A., \& SCHLAGHecken, F. (2002). Locus of inhibition in the masked priming of response alternatives. Journal of Motor Behavior, 34, 3-10.

Eriksen, C. W., Coles, M. G. H., Morris, L. R., \& O'Hara, W. P. (1985). An electromyographic examination of response competition. Bulletin of the Psychonomic Society, 23, 165-168.

Frame, K., Christie, J., \& Klein, R. (1993, November). Negative priming for spatial location: A methodological critique. Paper presented at the Annual Meeting of the Psychonomic Society, Washington, DC.

GuY, S., \& Buckolz, E. (2003, October). The locus of location repetition latency effects. Paper presented at the meeting of the Canadian Society for Psychology of Sport and Psychomotor Learning, Hamilton, ON.

Guy, S., Buckolz, E., \& Pratt, J. (2004). The influence of distractoronly prime trials on the location negative priming mechanism. Experimental Psychology, 54, 4-14.

Houghton, G., \& TipPER, S. P. (1994). A model of inhibitory mechanisms in selective attention. In D. Dagenbach \& T. Carr (Eds.), Inhibitory mechanisms in attention, memory, and language (pp. 53112). Orlando, FL: Academic Press.

KleIN, R. M., \& TAYLOR, T. L. (1994). Categories of cognitive inhibition with reference to attention. In D. Dagenbach \& T. H. Carr (Eds.), Inhibitory processes in attention, memory, and language (pp. 113150). San Diego: Academic Press.

Milliken, B., Joordens, S., Merikle, P., \& Seiffert, A. (1998). Selective attention: A reevaluation of the implications of negative priming. Psychological Review, 105, 203-229.

Milliken, B., Tipper, S. P., Houghton, G., \& Lupiáñez, J. (2000). Attending, ignoring, and repetition: On the relation between negative priming and inhibition of return. Perception \& Psychophysics, $\underline{62}_{2}$ 1280-1296.

MoORE, C. M. (1994). Negative priming depends on probe-trial conflict: Where has all the inhibition gone? Perception \& Psychophysics, 56, 133-147.

NEILL, W. T. (1997). Episodic retrieval in negative priming and repetition priming. Journal of Experimental Psychology: Learning, Memory, \& Cognition, 23, 1291-1305.

NeILl, W. T., Terry, K. M., \& VALDEs, L. A. (1994). Negative priming without probe selection. Psychonomic Bulletin \& Review, 1, 119-121.

Neill, W. T., Valdes, L. A., \& Terry, K. M. (1995). Selective attention and the inhibitory control of cognition. In F. M. Dempster \& C. J. Brainerd (Eds.), Interference and inhibition in cognition (pp. 207261). New York: Academic Press.

Smid, H., Mulder, G., \& Mulder, L. (1990). Selective response activation can begin before stimulus recognition is complete: A psychophysiological and error analysis of continuous flow. Acta Psychologica, 74, 169-201.

TIPPER, S. P. (1985). The negative priming effect: Inhibitory priming by ignored objects. Quarterly Journal of Experimental Psychology, 37A, 571-590.

TIPPER, S. P. (2001). Does negative priming reflect inhibitory mechanisms? A review and integration of conflicting views. Quarterly Journal of Experimental Psychology, 54A, 321-343.

TipPer, S. P., Brehaut, J. C., \& Driver, J. (1990). Selection of moving and static objects for the control of spatially directed action. Jour- 
nal of Experimental Psychology: Human Perception \& Performance, 16, $492-504$.

TipPer, S. P., \& CRANSTON, M. (1985). Selective attention and priming: Inhibitory and facilitatory effects of ignore primes. Quarterly Journal of Experimental Psychology, 37A, 581-611.

Tipper, S. P., Meegan, D., \& Howard, L. A. (2002). Action-centered negative priming: Evidence for reactive inhibition. Visual Cognition, 9, 591-614.

VÁLLE-InClán, F., \& Redondo, M. (1998). On the automaticity of ipsilateral response activation in the Simon effect. Psychophysiology, 35, 366-371.

\section{NOTE}

1. We have used an unbalanced design here in that not all possible target-distractor paired placements were employed (see Christie \& Klein, 2001). The use of unbalanced designs to keep trial numbers manageable is not uncommon (Tipper et al., 2002). The concern that unbalanced designs might interfere with the normal operation of the pro- cesses giving rise to the location NP effect because they could increase the predictability of probe-trial target location, and so also that of upcoming IR trials, is unwarranted. Contrasting balanced and unbalanced designs, Frame, Christie, and Klein (1993) as well as Guy, Buckolz, and Pratt (2004) have reported that the NP effect was unaltered with the use of an unbalanced design. Furthermore, Buckolz, Boulougouris, O'Donnell, and Pratt (2002) showed that increasing the predictability of probe-trial target location to as high as $75 \%$ validity left the NP effect unchanged. Unbalanced designs do not raise probe-trial location predictability to such high validity values, and so it is unlikely that such designs would disrupt the NP processes. Accordingly, it is improbable that the unbalanced procedure employed in this study produced aberrant findings. More direct assurance that this is the case comes from recent work that replicated the locus results observed here using a balanced design (Guy \& Buckolz, 2003).

(Manuscript received March 18, 2003; revision accepted for publication October 28, 2003.) 